\title{
Autophagy by Natural Products in Cancer Cells
}

\section{Thomas Efferth ${ }^{1 *}$ and Henry Johannes Greten ${ }^{2,3}$}

${ }^{1}$ Department of Pharmaceutical Biology, Institute of Pharmacy and Biochemistry, Johannes Gutenberg University, Staudinger Weg 5, 55128 Mainz, Germany

${ }^{2}$ Heidelberg school of Traditional Chinese Medicine, Heidelberg, Germany

${ }^{3}$ Biomedical sciences institute of Abel Sazar, University of Porto, Portugal

The physiological function of autophagy in normal cells is to maintain cellular homeostasis in nutrient-deprived states. The degradation of cytoplasmic material serves cell-autonomous energy production to promote cell survival. Hence, autophagy acts as prosurvival mechanism. Other autophagy-inducing metabolic stressors are growth-factor depriviation, hypoxia, and reactive oxygen species. Depending on the cellular context, autophagy also induces cell death. Therefore, autophagy has been described as type II-cell death, in contrast to apoptosis (type I-cell death). Cell death raised considerable interest in cancer pharmacology. Although natural products have been demonstrated to induce autophagy, the full therapeutic potential still needs to be explored.

Apoptosis has been one of the hottest topics in cell biology, immunology and pharmacology during the past two decades [13]. More recently, new forms of cell death were discovered such as necroptosis, immunogenic cell death, etc. Autophagy has also been described as a form of cell death, which can also act as pro-survival mechanism.

The term autophagy describes the degradation of intracellular cargo (mitochondria, proteins, other structures) within lysosomes. Three types of autophagy are known:

1) Macroautophagy is best analyzed as of yet, as is commonly termed "autophagy". Cargo delivery to lysosomes is mediated by vesicles with a membrane bi-layer. These vesicles ("autophagosomes") fuse with lysosomes ("autolysosome") to release intravesicular material.

2) Micro-Autophagy: Cytoplasmic material is taken up by lysosomes, by invagination of the lysosomal membrane.

3) Chaperone-mediated autophagy: Proteins form complexes with Hsc-70 or other chaperones, which are recognized by lysosomal associated membrane proteins (LAMP), and translocated into lysosomes.

Macroautophagy (hereafter, referred to as "autophagy" in this article) may be subclassified into "basal autophagy" (constitutive turnover of cytoplasmic components) and "induced autophagy" (degradation of intracellular cargo upon appropriate stimulation) [4].

Autophagy is described as type II -cell death in contrast to apoptosis (type I-cell death) [5], because it induces cell death depending on the cellular context in contrast to apoptosis (type I-cell death) [5]. Autophagy can precede, accompany or prevent apoptosis, and the molecular mechanisms of cross-talk between autophagy and apoptosis are still incompletely understood [6-11]. Autophagy and apoptosis may cooperate leading to cell death. In other cellular settings, autophagy may counteract apoptosis by creating a pro-survival cellular milieu. Finally, autophagy may enable apoptosis by providing energy to induce ATP-dependent apoptotic processes.

All forms of cell death have raised considerable interest in cancer pharmacology, since most if not all anticancer drugs induce apoptosis, or another form of cell death. Apoptosis represents an evolutionary very old mechanism of organisms to cope with toxic insults, either from environment (e.g. heavy metals) or from poisonous ingredients in foods or microbial infections. In clinical oncology, it has however, frequently been overseen that natural products from plants, fungi or microorganisms induce cell death and thus, provide a basis for the development for novel treatment strategies in cancer therapy. It has been well documented in the literature that natural products induce apoptosis in cancer cells $[12,13]$. Although, a number of papers also demonstrated that natural products induce autophagy (Table 1), the full therapeutic potential still needs to be explored [14]. Given the fundamental importance of autophagy in cell biology, and the fact that natural products represent a major resource for the development of cancer drugs [15], cytotoxic compounds from nature certainly deserve more attention in cancer therapy dealing with autophagy regulation.

\section{References}

1. Andersen MH, Becker JC, Straten PT (2005) Regulators of apoptosis: suitable targets for immune therapy of cancer. Nat Rev Drug Discov 4: 399-409.

2. Ocker M, Höpfner M (2012) Apoptosis-modulating drugs for improved cancer therapy. Eur Surg Res 48: 111-120.

3. Vyas VK, Chintha C, Pandya MR (2012) Biology and Medicinal Chemistry Approaches towards various apoptosis inducers. Anticancer Agents Med Chem.

4. Mizushima N (2007) Autophagy: process and function. Genes \& Dev 21: 2861 2873.

5. Schweichel JU, Merker HJ (1973) The morphology of various types of cell death in prenatal tissues. Teratology 7: 253-266.

6. Efferth T, Giaisi M, Merling A, Krammer PH, Li-Weber M (2007) The anti-malaria herbal compound artesunate triggers mitochondria-mediated apoptosis in leukemia T cells by inducing oxidative stress. PLoS ONE 2: e693.

7. Eisenberg-Lerner A, Bialik S, Simon H-U, Kimchi A (2009) Life and death partners: apoptosis, autophagy and the cross-talk between them. Cell Death Differ 16: 966-975.

8. Luo M, Liu X, Zu Y, Fu Y, Zhang S, et al. (2010) Cajanol, a novel anticancer agent from Pigeonpea [Cajanus cajan (L.) Millsp.] roots, induces apoptosis in human breast cancer cells through a ROS-mediated mitochondrial pathway. Chem Biol Interact 188: 151-160.

9. Hamacher-Brady A, Stein HA, Turschner S, Toegel I, Mora R, et al. (2011) Artesunate activates mitochondrial apoptosis in breast cancer cells via ironcatalyzed lysosomal reactive oxygen species production. J Biol Chem 286 6587-6601.

*Corresponding author: Thomas Efferth, Department of Pharmaceutical Biology, Institute of Pharmacy and Biochemistry, Johannes Gutenberg University, Staudinger Weg 5, 55128 Mainz, Germany, Tel: +49-6131-3925751; Fax: 496131-3923752; E-mail: efferth@uni-mainz.de

Received November 12, 2012; Accepted November 14, 2012; Published November 16, 2012

Citation: Efferth T (2012) Autophagy by Natural Products in Cancer Cells. Biochem Anal Biochem 1:e128. doi:10.4172/2161-1009.1000e128

Copyright: (c) 2012 Efferth T. This is an open-access article distributed under the terms of the Creative Commons Attribution License, which permits unrestricted use, distribution, and reproduction in any medium, provided the original author and source are credited. 
10. Reichert S, Reinboldt V, Hehlgans S, Efferth T, Rödel C, et al. (2012) A radiosensitizing effect of artesunate in glioblastoma cells is associated with a diminished expression of the inhibitor of apoptosis protein survivin. Radiother Oncol 103: 394-401.

11. Zhang Y, Luo M, Zu Y, Fu Y, Gu C, et al. (2012) Dryofragin, a phloroglucinol derivative, induces apoptosis in human breast cancer MCF-7 cells through ROS-mediated mitochondrial pathway. Chem-Biol Interact 199: 129-136.

12. Efferth T (2007) Willmar Schwabe Award 2006: antiplasmodial and antitumor activity of artemisinin - from bench to bedside. Planta Med 73: 299-309.

13. Fulda S (2010) Modulation of apoptosis by natural products for cancer therapy Planta Med 76: 1075-1079.

14. Zhang X, Chen LX, Ouyang L, Cheng Y, Liu B (2012) Plant natural compounds: targeting pathways of autophagy as anti-cancer therapeutic agents. Cell Prolif 45: $466-476$

15. Newman DJ, Cragg GM (2012) Natural products as sources of new drugs over the 30 years from 1981 to 2010 . J Nat Prod 75: 311-335.

16. Szeto J, Kaniuk NA, Canadien V, Nisman R, Mizushima N, et al. (2006) ALIS are stress-induced protein storage compartments for substrates of the proteasome and autophagy. Autophagy 2: 189-199.

17. Pedro M, Lourenço CF, Cidade H, Kijjoa A, Pinto M, et al. (2006) Effects of natural prenylated flavones in the phenotypical ER (+) MCF-7 and ER (-) MDAMB-231 human breast cancer cells. Toxicol Lett 164: 24-36

18. Mori S, Sawada T, Okada T, Ohsawa T, Adachi M, et al. (2007) New antiproliferative agent, MK615, from Japanese apricot "Prunus mume" induces striking autophagy in colon cancer cells in vitro. World J Gastroenterol 13 6512-6517.

19. Lin HM, Tseng HC, Wang CJ, Chyau CC, Liao KK, et al. (2007) Induction of autophagy and apoptosis by the extract of Solanum nigrum Linn in HepG2 cells. J Agric Food Chem 55: 3620-3628.

20. Delmulle L, Vanden Berghe T, Keukeleire DD, Vandenabeele P (2008) Treatment of PC-3 and DU145 prostate cancer cells by prenylflavonoids from hop (Humulus lupulus L.) induces a caspase-independent form of cell death. Phytother Res 22: 197-203.

21. Meschini S, Condello M, Calcabrini A, Marra M, Formisano G, et al. (2008) The plant alkaloid voacamine induces apoptosis-independent autophagic cell death on both sensitive and multidrug resistant human osteosarcoma cells. Autophagy 4: 1020-1033.

22. Lin MH, Liu SY, Liu YC (2008) Autophagy induction by a natural ingredient of areca nut. Autophagy 4: 967-968.

23. Liu SY, Lin MH, Hsu YR, Shih YY, Chiang WF, et al. (2008) Arecoline and the 30-100 kDa fraction of areca nut extract differentially regulate mTOR and respectively induce apoptosis and autophagy: a pilot study. J Biomed Sci 15 823-831.

24. Yang J, Wu LJ, Tashino SI, Onodera S, Ikejima T (2008) Reactive oxygen species and nitric oxide regulate mitochondria-dependent apoptosis and autophagy in evodiamine-treated human cervix carcinoma HeLa cells. Free Radic Res 42: 492-504.

25. Bredholt T, Dimba EA, Hagland HR, Wergeland L, Skavland J, et al. (2009) Camptothecin and khat (Catha edulis Forsk.) induced distinct cell death phenotypes involving modulation of $\mathrm{C}-\mathrm{FLIP}_{\mathrm{L}}, \mathrm{Mcl}-1$, procaspase-8 and mitochondrial function in acute myeloid leukemia cell lines. Mol Cancer 8: 101.

26. King FW, Fong S, Griffin C, Shoemaker M, Staub R, et al. (2009) Timosaponin Alll is preferentially cytotoxic to tumor cells through inhibition of mTOR and induction of ER stress. PLOS. ONE 4: e7283.

27. Stander A, Marais S, Stivaktas V, Vorster C, Albrecht C, et al. (2009) In vitro effects of Sutherlandia frutescens water extracts on cell numbers, morphology, cell cycle progression and cell death in a tumorigenic and a non-tumorigenic epithelial breast cell line. J Ethnopharmacol 124: 45-60.

28. Juncker T, Schumacher M, Dicato M, Diederich M (2009) UNBS1450 from Calotropis procera as a regulator of signaling pathways involved in proliferation and cell death. Biochem Pharmacol 78: 1-10.

29. Brunelli E, Pinton G, Bellini P, Minassi A, Appendino G, et al. (2009) Flavonoidinduced autophagy in hormone sensitive breast cancer cells. Fitoterapia 80 : 327-332.

30. Lei HY, Chang CP (2009) Lectin of Concanavalin A as an anti-hepatoma therapeutic agent. J Biomed Sci 16:10.

31. Law BYK, Wang M, Ma DL, Al-Mousa F, Michelangeli F, et al. (2010) Aliso $\mathrm{B}$, a novel inhibitor of the sarcoplasmic/endoplasmic reticulum $\mathrm{Ca}(2+)$ ATPase pump, induces autophagy, endoplasmic reticulum stress, and apoptosis. Mo Cancer Ther 9: 718-730.

32. Ooi KL, Muhammad TST, Sulaiman SF (2010) Growth arrest and induction of apoptotic and non-apoptotic programmed cell death by, Physalis minima L. chloroform extract in human ovarian carcinoma Caov-3 cells. J Ethnopharmacol 128: $92-99$.

33. Carr G, Williams DE, Díaz-Marrero AR, Patrick BO, Bottriell H, et al. (2010) Bafilomycins produced in culture by Streptomyces spp. isolated from marine habitats are potent inhibitors of autophagy. J Nat Prod 73: 422-427.

34. Thyagarajan A, Jedinak A, Nguyen H, Terry C, Baldridge LA, et al. (2010) Triterpenes from Ganoderma lucidum induce autophagy in colon cance through the inhibition of p38 mitogen-activated kinase (p38 MAPK). Nutr Cancer 62: 630-640

\begin{tabular}{|c|c|c|c|c|}
\hline Compound/agent & Species & Cell line & Autophagic markers & Reference \\
\hline puromycin & & $\begin{array}{l}\text { HeLa cervix cancer, DU145 } \\
\text { prostate cancer, RAW264.7 } \\
\text { macrophages }\end{array}$ & $\begin{array}{l}\text { formation of polyubiquitinated defective ribosomal products } \\
\text { (DRiPs) } \uparrow\end{array}$ & {$[16]$} \\
\hline prenylated flavones & & $\begin{array}{l}\mathrm{ER}(+) \text { and MCR-7 } \\
\text { ER(-) MDA-MB-23I } \\
\text { breast cancer }\end{array}$ & $\begin{array}{l}\text { autophagic vacuolization } \\
\text { monodansylcadaverine staining (=autophagosome-specific dye) }\end{array}$ & {$[17]$} \\
\hline MK615 extract & $\begin{array}{l}\text { Prunus mume (Japanese } \\
\text { apricot) }\end{array}$ & $\begin{array}{l}\text { SW } 480, \text { CoLo, WiDr } \\
\text { Colon Ca }\end{array}$ & autophagic vacuoles, ATG8 immuno-fluorescence [apoptosis] & [18] \\
\hline extract & Solanum nigrum $\mathrm{L}$. & $\begin{array}{l}\text { HepG2 } \\
\text { liver cancer }\end{array}$ & $\begin{array}{l}\text { low doses: autophagic vacuoles, LC-I and LC-II expression } \\
\uparrow, \text { Bcl- } 2 \text { and Akt expression } \downarrow \text { high doses: p-JNK and Bax } \\
\text { expression } \uparrow \text { apoptosis }\end{array}$ & [19] \\
\hline $\begin{array}{l}\text { xanthohumol, } \\
\text { isoxanthohumol, } \\
\text { 8-prenylnaringenin, } \\
\text { 6-prenylnaringenin }\end{array}$ & Humulus lupulus L. & PC-3, Du 145 prostate cancer & $\begin{array}{l}\text { autophagic vacuoles, } \\
\text { no caspase- } 3 \text { activation, no apoptosis }\end{array}$ & {$[20]$} \\
\hline voacamine & Peschiera fuchsiaefolia & $\begin{array}{l}\text { Multidrug-resistant } \\
\text { osteosarcoma }\end{array}$ & $\begin{array}{l}\text { autophagic vacuolization, LC-3 expression and conversion, } \\
\text { monodansylcadaverine staining }\end{array}$ & [21] \\
\hline proteoglycan extract & areca nut & CE81T/VGH cells & autophagic vacuoles, acidic vesicles, LC3-I cleavage & [22] \\
\hline 30-100 kDA extract & areca nut & & $\begin{array}{l}\text { autophagic vacuoles, acidic vesicles, phosphorylation of mTOR } \\
\downarrow, \text { LC-I cleavage }\end{array}$ & [23] \\
\hline evodiamine & Tetradium spec. & HeLa cervix cancer & $\begin{array}{l}\text { monodansylcadaverine staining, Beclin-I expression } \uparrow, \text { LC3 } \\
\text { expression } \uparrow, 3-\mathrm{MA}(=\text { autophagy inhibitor }) \text { decreased cell viability }\end{array}$ & [24] \\
\hline
\end{tabular}


Page 3 of 3

\begin{tabular}{|c|c|c|c|c|}
\hline organic extract & Catha edulis Forsk. & Leukemia cell lines MOLM-13 & $\begin{array}{l}\text { morphological features of autophagy apoptosis: McR- } 1 \downarrow \text {, c-FLIPL } \\
\text { cleavage } \uparrow \text {, pro-caspase- } 8 \text { activation } \uparrow ; \text { mitochondrial damage }\end{array}$ & {$[25]$} \\
\hline timasaponin A III & $\begin{array}{l}\text { Anemarrhena } \\
\text { asphodeloides }\end{array}$ & various cancer cell lines & $\begin{array}{l}\text { mTORC1 inhibition induction of endoplasmic reticulum stress } \\
(\text { elF2alpha phosphorylation } \uparrow ; \text { caspase- } 4 \text { activation } \uparrow) \rightarrow \text { pro- } \\
\text { apoptotic pathways }\end{array}$ & [26] \\
\hline extract & Sutherlandia frutescens & MCF-7 breast cancer & $\begin{array}{l}\text { autophagic vacuolarization hypercondensed } \\
\text { chromatin } \rightarrow \text { apoptosis }\end{array}$ & [27] \\
\hline $\begin{array}{l}\text { UNBS } 145001 \\
\text { (=derivative of } \\
\text { 2-oxovoruscharin } 02 \text { ) }\end{array}$ & Calotropis procera & & sodium pump inhibitor, disorganization of actin cytoskeleton & [28] \\
\hline isocannflavin B & & T47D & $\begin{array}{l}\text { autophagic vacuolization, Akt phophorylation } \downarrow \text {; p21 (Cip21) } \\
\text { expression } \uparrow\end{array}$ & [29] \\
\hline concanavalin A & Jack bean seeds & & autophagy, checken immunomodulation & {$[30]$} \\
\hline alisol B & Alisma orientale (rhizome) & various cancer cell lines & $\begin{array}{l}\text { autophagosome flux formation; cell death, } \mathrm{G}_{\mathrm{o}} / \mathrm{G}_{1} \text { cell cycle arrest; } \\
\text { activation of CaMMK-AMPK-mTOR pathway; disruption of } \mathrm{Ca}^{2+} \\
\text { homeostasis; binding to SERCA }\end{array}$ & [31] \\
\hline chloroform extract & Physalis minima L. & Caov-3 & Human ovarian cancer vacuolated morphology; [apoptosis] & [32] \\
\hline bafilomycin $\mathrm{F}, \mathrm{G}, \mathrm{H}$, and $\mathrm{J}$ & Streptomyces ssp. & MCF-7 breast cancer & microscopy and biochemical assays & [33] \\
\hline triterpene extract & Ganoderma lucidum & Colon cancer & 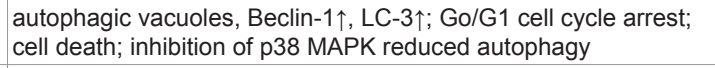 & [34] \\
\hline artesunate & Artemisia annua & MCF-7 breast cancer & $\begin{array}{l}\text { endolysosomal and autophagosomal compartments, inhibiting } \\
\text { autophagosome turnover and causing perinuclear clustering of } \\
\text { autophagosomes, early and late endosomes, and lysosomes. }\end{array}$ & [9] \\
\hline
\end{tabular}

Table 1: Natural products inducing autophagy in cancer cells. 